\title{
Purging of density stabilized basins
}

\author{
S. W. ARMFIELD \\ Centre for Water Research, Department of Civil and Environmental Engineering, \\ University of Western Australia, Nedlands, WA 6009, Australia \\ and \\ W. DEBLER \\ Mechanical Engineering and Applied Mechanics, University of Michigan, Ann Arbor, \\ MI 48109, U.S.A.
}

(Received 3 May 1991 and in final form 16 August 1991)

\begin{abstract}
A combined laboratory and numerical investigation of the purging of density stabilized basins has been undertaken. The laboratory investigation was carried out in a flume into which a false floor containing a cavity filled with dense fluid was set. The fluid in the cavity was then set into motion and ultimately purged by a fresh water overflow. The experiment was simulated numerically by a direct finite volume solution of the Navier-Stokes equations plus solute transport equation using the Boussinesq buoyancy approximation. The experimental results have been used to validate the numerical method, and a number of flow features have been identified. These include a large amplitude internal wave, a travelling wave and a vortex structure in the cavity. All these features contribute to the efflux of dense fluid from the cavity.
\end{abstract}

\section{INTRODUCTION}

UNDERSTANDING the mechanics of purging of density stabilized ponds by a lighter overflow is important in a number of areas of geophysical fluid mechanics. For instance, it is common, in regions of annual flow and relatively saline water tables, for pools of salt water to accumulate in the beds of rivers. During low flow periods the water table will saturate the pools in the river bed, and the small river flow will be unable to purge the denser fluid. A similar phenomenon can occur at river mouths and in estuaries where tidal intrusions can provide the saline water that fills the irregularities in the river bed.

The fluid forming such saline pools in the inland waters of Australia is also typically hypoxic, rendering them uninhabitable by fish and other aerobic organisms; hypoxia renders bed substrates and organic debris inaccessible for cover, feeding or resting, and probably reduces secondary production [1]. Understanding the fluid mechanics involved in the purging of such pools is therefore an important factor in the development of a river management strategy.

In the present investigation a combined numerical and experimental study has been undertaken as a first step towards developing an understanding of the fluid mechanics involved in the purging process. It is anticipated that the numerical model will be the primary investigative tool, with the experimental results being used to validate the numerical results at a number of points in the solution domain in order to maximize the range of parameters studied.

The numerical model solves the Navier-Stokes equations plus the solute transport equation in two dimensions using a finite-volume scheme, described in
Section 2. The model, at its present stage of development, is suitable only for laminar flows and for pools in the form of rectangular cavities.

The experimental apparatus, also described in Section 2, consists of a laboratory flume modified with a false floor into which a transparent box was fitted creating a cavity, the liquid contents of which could be set in motion by the channel flow. Initially, salt water was in the basin and the fresh water in the channel was abruptly set into motion. Unfortunately the construction of the flume was such that it was impossible to obtain a low Reynolds number laminar flow for direct comparison with the numerical model, and thus comparison of the small scale motion at this stage would be inappropriate. None the less a qualitative comparison indicates that the model accurately predicts the large scale features of the flow, which for the early part of the development may be summarized as follows.

(1) The overflow initially pushes out a large splash of the denser cavity fluid.

(2) Vorticity shed from the upstream corner of the cavity forms a vortex which pulls lighter fluid into the cavity and mixes it with the denser fluid there, with an accompanying secondary splash.

(3) A continuing seiching in the cavity ejects fluid from the resulting intermediate density layer.

(4) The development of a circulatory motion in the upper portion of the cavity slowly transports the denser liquid below it by shear stresses and molecular diffusion.

Stages (1), (2) and (3) occur very rapidly, with (4) taking typically ten times as long. 


\section{NOMENCLATURE}

$F r \quad$ Froude number

$g$ acceleration due to gravity

$h_{\mathrm{c}}$ height of cavity

$H$ height of duct

$k$ internal wavenumber

$p \quad$ period of seiche

$P$ pressure

$P r \quad$ Prandtl number, $v / \kappa$

$R a$ Rayleigh number, $g H^{3}(\Delta \rho) / v k$

Re Reynolds number

$t$ time

$U \quad x$ velocity component

$\bar{U}$ mean entrance velocity

$V \quad y$ velocity component $x$ horizontal distance from bottom left corner of duct

$y \quad$ vertical distance form bottom left corner of duct.

\section{Greek symbols}

$\delta \quad$ interface drop

$\boldsymbol{\kappa} \quad$ thermal diffusivity

$\lambda$ internal wavelength

$v \quad$ kinematic viscosity

$\rho$ density

$\rho_{c} \quad$ initial density in cavity

$\rho_{\mathrm{d}} \quad$ initial density in duct

$\Delta \rho$ initial density variation, $\left(\rho_{\mathrm{c}}-\rho_{\mathrm{d}}\right) / \rho_{\mathrm{d}}$

$\sigma \quad$ solute.
In the remainder of the paper the numerical and experimental methods are presented in Section 2. The results are presented in Section 3, with a discussion in Section 4 and the conclusions in Section 5.

\section{NUMERICAL AND EXPERIMENTAL METHODS}

\subsection{Governing equations}

The governing equations are the Navier-Stokes equations and the solute transport equation, which are expressed in incompressible and non-dimensional form in euclidean coordinates as follows:

$$
\begin{aligned}
& U_{1}+U U_{x}+V U_{y}=-P_{x}+\frac{1}{R e}\left(U_{x x}+U_{y y}\right) \\
& V_{1}+U V_{x}+V V_{y}=-P_{y}+\frac{1}{R e}\left(V_{x x}+V_{y y}\right)-\frac{R a \sigma}{P r R e^{2}} \\
& U_{x}+V_{y}=0 \\
& \sigma_{r}+U \sigma_{x}+V \sigma_{y}=\frac{1}{R e P r}\left(\sigma_{x x}+\sigma_{y y}\right)
\end{aligned}
$$

where subscripts indicate partial differentiation.
The relation between the solute concentration $\sigma$ and the density $\rho$ is $\rho=\rho_{\text {walcr }}+\sigma$. In the above equations length is non-dimensionalized by $H$, the height of the duct, average velocity by $\bar{U}$, time by $H / \bar{U}$ and the solute by $\sigma_{\text {cavily }}-\sigma_{\text {ducl }}$.

\subsection{Discretization}

The domain and distribution of points are shown in Fig. 1. As can be seen, the domain consists of a square cavity, initially containing the denser fluid, beneath a rectangular duct, initially containing the lighter fluid. The boundary consists of the free surface, the inlet at the left hand end of the duct, the exit at the right hand end, and the bottom. A non-uniform discretization on a rectangular grid is used to place the greatest resolution in the boundary layer regions and at the cavity-duct interface. Variables are then stored at nodal points on a non-staggered grid. The index $i$ is used to indicate location in the $x$ direction and $j$ indicates location in the $y$ direction. Values of variables at the nodal point $(i, j)$ are given as, for instance $x^{i}, y^{j}, U^{i j}$ and so on. As a finite-volume method is used no nodes lie on the boundaries. Boundary values are set by including an extra point outside the boundary with a prescribed value. The value at

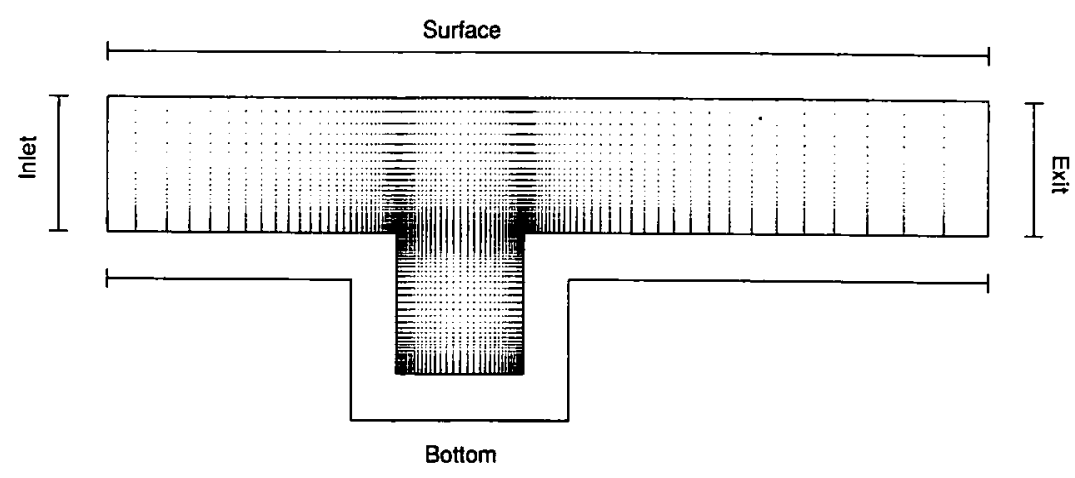

Fig. 1. Computational domain with discretization. 
the boundary is then the average of that point and the nearest point in the domain. When values of dependent variables are required at other than nodal points they are obtained by linear interpolation.

Finite volumes are used to convert differential terms in the governing equations in the following way. All second derivatives are approximated by second-order central differences as

$$
\begin{aligned}
U_{x . x}\left(x^{i}, y^{j}\right)= & \left(\frac{U^{i+1}-U^{i}}{\Delta x^{i+1}}-\frac{U^{i}-U^{i-1}}{\Delta x^{i}}\right)^{\prime} / \\
& \left(\frac{\Delta x^{i+1}+\Delta x^{i}}{2}\right)+O\left(\Delta x^{2}\right)=S D U_{j}^{i}
\end{aligned}
$$

where $\Delta x^{2}=\Delta x \times \Delta x, \Delta x^{i}=x^{i}-x^{i-1}$ and $S D$ is a finite difference operator.

Derivatives occurring in convective terms are approximated using a third-order upwinded and bounded scheme [2], as follows:

$$
U U_{x}\left(x^{i}, y^{j}\right)=\left(F^{i+1 ; 2, j}-F^{i-1 ; 2 . j}\right) 2 /\left(\Delta x^{i+1}+\Delta x^{i}\right)
$$

where

$$
F^{i+1 / 2, j}=U^{i+1 / 2}\left(U^{i+1 / 2}-S D U^{i j}\left(\Delta x^{i+1}\right)^{2}(1 / 8+\alpha)\right)
$$

assuming $U^{i+1 / 2, j}$ is positive. $\alpha$ is then obtained as

$$
\alpha=\left[\frac{\hat{U}^{i+1 / 2}-3 / 8\left(2 \hat{U}^{i}+1\right)}{2 \hat{U}^{i}-1}\right]
$$

with

$$
\hat{U}^{i}=\frac{U^{i}-U^{i-1}}{U^{i+1}-U^{i-1}}
$$

and

$$
\hat{U}^{i+1 / 2}=\left\{\begin{array}{lll}
\hat{U}^{i} & \text { if } & \hat{U}^{i} \notin[0,1] \\
3 \hat{U}^{i} & \text { if } & \hat{U}^{i} \in[0,1 / 6] \\
1 & \text { if } & \hat{U}^{i} \in[5 / 6,1] \\
3 / 8\left(2 \hat{U}^{i}+1\right) & \text { if } & \hat{U}^{i} \in[1 / 6,5 / 6] .
\end{array}\right.
$$

The use of the bounded scheme, which is seen to be a modification of the well known QUICK scheme [3], has been found to be essential for obtaining high Reynolds number simulations of the flow. When a conventional third-order upwinding scheme, such as QUICK, was used it resulted in severe oscillations at the interface, which ultimately caused the solution to collapse. This is believed to be due primarily to the high flow to grid skewness present in this flow that occurs as the interface is distorted.

\subsection{Integration}

The above equations are integrated numerically in the following way. All variables are known at time step $t^{\prime \prime}$. First an initial estimate for $(U, V)^{n+1}$ is obtained, denoted as $(U, V)^{n+1, m}$ with $m=0$, from

$$
\begin{aligned}
& G 1\left(U^{n+1, m}\right)=G 2\left(U^{n}\right)-\left(P^{i+1}-P^{i-1}\right)^{j, n+1 / 2 . m} / \\
& \left(\Delta x^{i+1}+\Delta x^{i}\right) \\
& G 1\left(V^{n+1, m}\right)=G 2\left(V^{n}\right)-\left(P^{i+1}-P^{j-1}\right)^{i, n+1 / 2, m} /
\end{aligned}
$$$$
\left(\Delta y^{j+1}+\Delta y^{j}\right) \text {. }
$$

$G 1=(L / 2+1 / \Delta t), G 2=(-L / 2+1 / \Delta t)$ and $L$ is in discrete form a block quintadiagonal matrix with components obtaincd from the differencing given above which is inverted using an alternating direction implicit scheme. The best available guess is used for $P^{n+1 / 2,0}$. Next a correction for $P$ is obtained by solving the following Poisson equation for $P c$, the pressure correction:

$$
\begin{aligned}
& {\left[g^{i+1 / 2}\left(\frac{P c^{i+1}-P c^{i}}{\Delta x^{i+1}}\right)-g^{i-1 / 2}\left(\frac{P c^{i}-P c^{i-1}}{\Delta x^{i}}\right)\right]^{j} 2 /} \\
& \left(\Delta x^{i+1}+\Delta x^{i}\right)+\left[g^{j+1 / 2}\left(\frac{P c^{j+1}-P c^{j}}{\Delta y^{j+1}}\right)\right. \\
& \left.-g^{j-1 / 2}\left(\frac{P c^{j}-P c^{j-1}}{\Delta y^{j}}\right)\right]^{i} 2 /\left(\Lambda y^{j+1}+\Lambda y^{j}\right) \\
& =\left(\tilde{U}^{i+1 / 2}-\tilde{U}^{i-1 / 2}\right)^{j \cdot n+1 . m} 2 /\left(\Lambda x^{j+1}+\Lambda x^{j}\right) \\
& +\left(\tilde{V}^{j+1: 2}-\tilde{V}^{j-1 / 2}\right)^{i, n+1 . m} 2 /\left(\Delta y^{j+1}+\Delta y^{j}\right)
\end{aligned}
$$

where $g$ is the inverse of the diagonal of $G 1$. As can be seen, this is a finite-volume discretization about the point $(i, j)$ consisting of, in the $x$ direction for example, the difference of the terms $g(P c)_{x}$ and $\tilde{U}$ at the points $x=x^{i+1 / 2}, x=x^{i-1 / 2}$, and similarly for the $y$ terms.

$P^{n+1 / 2, m}, U^{n+1 . m}, V^{n+1 . m}$ are then corrected as

$U^{i, j, n+1, m+1}=U^{i, j, n+1, m}-g^{i}\left(P c^{i+1}-P c^{i-1}\right) /$

$$
\left(\Delta x^{i+1}+\Delta x^{i}\right)
$$

$V^{i, j, n+1, m+1}=V^{i, j, n+1, m}-g^{j}\left(P c^{j+1}-P c^{j-1}\right) /$

$$
\begin{aligned}
\left(\Delta y^{j+1}+\Delta y^{j}\right) \\
P^{i, j, n+1 / 2 . m+1}=\operatorname{Rr} P c^{i, j}-P^{i, j, n+1 / 2, m}
\end{aligned}
$$

with $R x$ an under-relaxation factor for the pressure, which for the present simulation was set to 0.6 .

The terms $\tilde{U}$ and $\tilde{V}$ are interpolated velocities defined in the following way:

$$
\begin{aligned}
& \tilde{U}^{i+1 / 2 . j}=\left(U^{i+1}+U^{i}\right)^{j} / 2+R^{i+1 / 2 j} \\
& \tilde{V}^{j+1 / 2 . i}=\left(V^{j+1}+V^{j}\right)^{i} / 2+R^{j+1 / 2, i} .
\end{aligned}
$$

The $R$ terms are then defined to be

$$
\begin{aligned}
R^{i+1 / 2, j} & =\left[\left(g^{i+1}\left(\frac{P^{i+2}-P^{i}}{\Delta x^{i+2}+\Delta x^{i+1}}\right)\right.\right. \\
& \left.\left.+g^{i}\left(\frac{P^{i+1}-P^{i-1}}{\Delta x^{i+1}+\Delta x^{i}}\right)\right) / 2-g^{i+1 / 2}\left(\frac{P^{i+1}-P^{i}}{\Delta x^{i+1}}\right)\right]^{j} \\
R^{j+1 / 2 . i} & =\left[\left(g^{i+1}\left(\frac{P^{j+2}-P^{j}}{\Delta y^{j+2}+\Delta y^{j+1}}\right)\right.\right. \\
+ & \left.\left.g^{j}\left(\frac{P^{j+1}-P^{j}}{\Delta y^{j+1}+\Delta y^{j}}\right)\right) / 2-g^{j+1 / 2}\left(\frac{P^{j+1}-P^{j}}{\Delta y^{j+1}}\right)\right]^{i} .
\end{aligned}
$$

The $R$ terms as defined above ensure the discretization is strongly elliptic and allow the non-staggered mesh to be used. Without these terms the discrete scheme is non-elliptic and its use will result in a grid scale 
oscillation of the pressure ficld and subsequent breakdown of the solution. The use of a non-staggered scheme, as compared to a conventional staggered scheme, where the discrete ellipticity requirement is automatically satisfied, leads to an improved efficiency in coding and running due to the fact that operators that are identical in continuous form are then identical in discrete form [4].

\subsection{Boundary and initial values}

At the inlet a parabolic velocity profile is specified while zero downstream variation is assumed at the outlet. The bottom is non-slip and the surface is zero shear. For the solute the surface, bottom and outlet all have zero normal gradient while at the inlet the value is set to zero. The normal derivative of the pressure correction is set to zero everywhere on the boundary, while the pressure, which is obtained from the pressure correction at all interior points, is obtained on the boundary using a second-order extrapolation from the interior points. At the interior corners a separate extrapolated pressure is obtained for the pressure used in the $x$-derivative at the adjacent exterior $x$-node and for the pressure used in the $y$ derivative at the adjacent exterior $y$-node. Initially the fluid is at rest everywhere. The fluid in the cavity is at a non-dimensional solute concentration of 1 while that in the channel is at a concentration of 0 . At $t=0$ the parabolic velocity profile is switched on at the entrance and the flow is allowed to develop.

\subsection{Experimental method}

A laboratory flume $11 \mathrm{~m}$ long was modified with a false floor to create a duct of cross-section $10 \mathrm{~cm}$ high and $29 \mathrm{~cm}$ wide. A square cavity of dimension 11.6 $\mathrm{cm} \times 11.6 \mathrm{~cm}$ was set into the false floor of the flume and filled with salt water at a predetermined density with food colouring at a concentration of about 2 parts per thousand mixed in as a marker, while the duct was filled with fresh water. The experiment was then left to stand for $2 \mathrm{~h}$ to allow any circulation to diffuse out.

At time $t=0$, water was admitted to the flume through a flow straightener and turbulence damper, setting in motion the upper layer and ultimately purging thereby the dense fluid in the cavity. During the development of the flow photographs were taken at regular intervals, with the marker added to the dense fluid acting to make the purging process visible.

\section{RESULTS}

Numerical results have been obtained for Reynolds number-Rayleigh number pairs and corresponding density variation values of

(1) $R e=700, R a=8 \times 10^{6}, \Delta \rho=1 \times 10^{-5}$

(2) $R e=350, R a=8 \times 10^{6}, \Delta \rho=1 \times 10^{-5}$

(3) $R e=350, R a=4 \times 10^{6}, \Delta \rho=5 \times 10^{-6}$

(4) $R e=175, R a=8 \times 10^{6}, \Delta \rho=1 \times 10^{-5}$
(5) $R e=10000, R u=3.75 \times 10^{10}, \Delta \rho=0.005$

(6) $R e=10000, R a=2.2 \times 10^{10}, \Delta \rho=0.003$

with the solute assumed to be salt and thus the Prandtl number, $\mathrm{Pr}$, set to 750 .

The internal Froude number based on the cavity height may be obtained from the relation

$$
F r=\left(\frac{R e^{2} P r}{R a h_{\mathrm{c}}}\right)^{1 / 2}
$$

where $h_{\mathrm{c}}=1.16$ is the height of the cavity non-dimensionalized by $H$, the duct height. Froude numbers for each of the cases are then obtained as
(1) $\mathrm{Fr}=6.2$
(2) $F r=3.1$
(3) $F r=4.4$
(4) $F^{r}=1.6$
(5) $F r=1.31$
(6) $F_{r}=1.71$.

The parameter values for cases (1)-(4) were chosen to give a range of Froude numbers all greater than one, but with Reynolds numbers low enough to correspond to a laminar flow regime. Cases (5) and (6) were chosen to correspond to the experimental data.

The results are presented in dimensional form, based on a duct height of $10 \mathrm{~cm}$ and a square cavity of $11.6 \times 11.6 \mathrm{~cm}^{2}$, for velocity time series. Nusselt number time series, density contours and stream function contours. Thus for case (1) $R e=700$ corresponds to an inlet velocity of $7 \mathrm{~mm} \mathrm{~s}^{-1}$, and for case (2) $R e=10000$ corresponds to an inlet velocity of $10 \mathrm{~cm}$ $\mathrm{s}^{-1}$. As the velocity variation in the duct is an orderof-magnitude greater than that in the cavity it has been necessary to set upper and lower bounds to the stream function contours plotted to make clear the flow structure in the cavity, effectively removing the streamlines representing the flow in the duct from the figures.

Figures 2(a) and (b) show the density contours and streamlines at $t=21 \mathrm{~s}$ after initiation of the flow for case (1). At this time a small amount of denser fluid has been pushed out of the cavity by the overflow, while at the inlet corner of the cavity the interface has been pushed down a corresponding amount, with a small crest visible projecting in the downstream direction. The streamlines show that a vortex has formed centered just downstream of the cavity inlet corner. Evidently the crest observed in the density contours consists of dense fluid that is being rolled up by the vortex. As is àlso seen, a number of streamlines from the duct overflow enter and traverse the cavity, exiting at the downstream corner. It is these streamlines that produce the splash of fluid observed in the density contours.

Figure 3(a) shows the density contours at $t=66 \mathrm{~s}$ after initiation. The splash of denser fluid has now completely exited the cavity and is being transported to the exit by the overflow, while the crest initially located at the inlet corner has moved across the cavity 


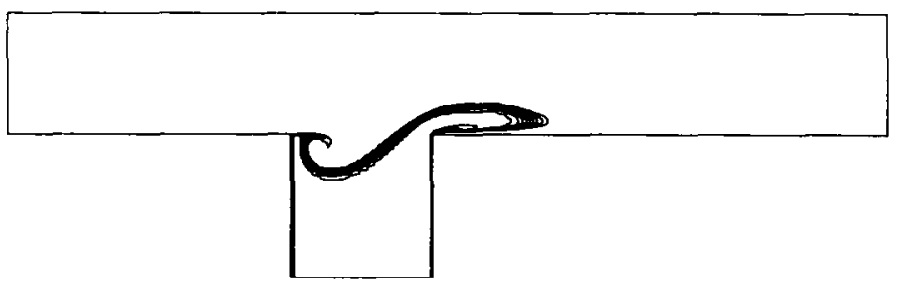

Fig. 2(a). Density contours for case (1) at $t=21 \mathrm{~s}$.

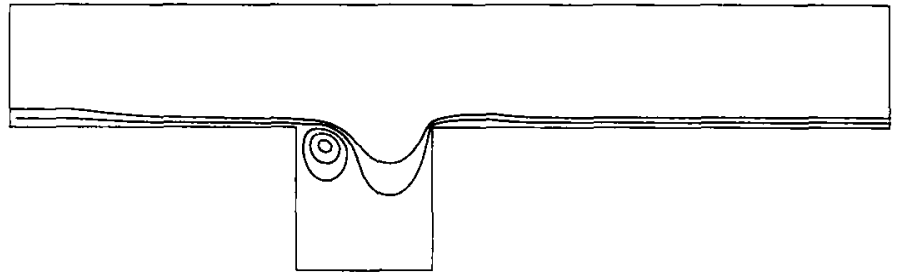

FIG. 2(b). Stream function contours for case (1) at $t-21 \mathrm{~s}$.

until it has almost reached the exit wall. At the same lime the crest has grown considerably in amplitude and continued to roll up. The streamlines at $t=66 \mathrm{~s}$ (Fig. 3(b)) show that the small vortex located at the cavity entrance has now grown to encompass almost the entire cavity, with the centre located just past the cavity centreline. Once again it is clear that the crest of denser fluid is being rolled up by the vortex. No streamlines from the overflow traverse the cavity at this stage, consistent with the observation above that the splash has completely exited the cavity.

At $t=133 \mathrm{~s}$ after initiation (Figs. 4(a) and (b)), the crest has hit the cavity exit corner, with a substantial part of it splashing out as a result. The remainder of the crest, together with an enveloped volume of fluid from the light overflow, has been pulled down into the cavity, forming a large region of instability. The streamlines indicate that the enveloped volume of light nuid is located at the centre of the vortex, which still encompasses almost the entire cavity.

By $876 \mathrm{~s}$ after initiation (Figs. 5(a) and (b)) a strongly mixing region is seen to have formed in the upper part of the cavity, with a particular concentration of mixing near to the exit wall. The interface between the mixing region and the unmixed denser fluid has been pushed down to approximately half the height of the cavity. The streamlines show that the vortex is now confined to the mixing region. with its centre located near to the exit wall, while a weak counter-rotating vortex has formed in the lower unmixed part of the cavity.

Results obtained for cases (2), (3) and (4), which are not shown, indicate a similar development; that is, the formation of the initial splash and a crest that

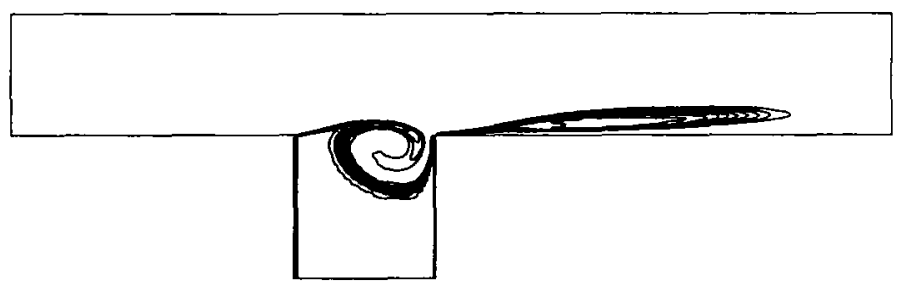

FIG. 3(a). Density contours for case (1) at $t=66 \mathrm{~s}$.

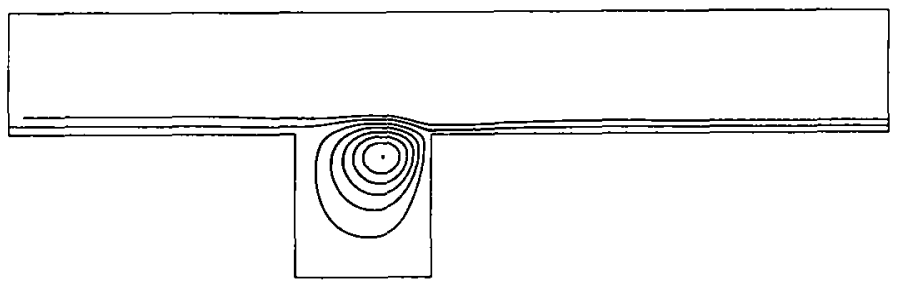

FIG. 3(b). Stream function contours for case (I) at $t=66 \mathrm{~s}$. 


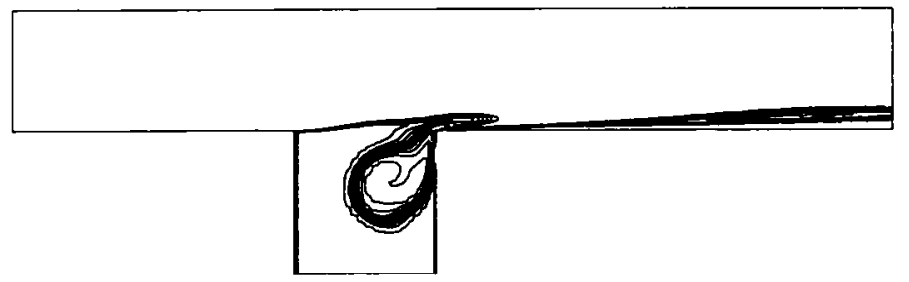

FIG. 4(a). Density contours for case (1) at $t=133 \mathrm{~s}$.

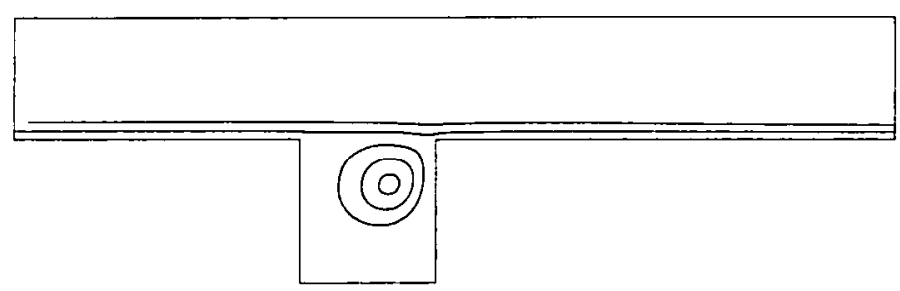

FIG. 4(b). Stream function contours for case (1) at $t=133 \mathrm{~s}$.

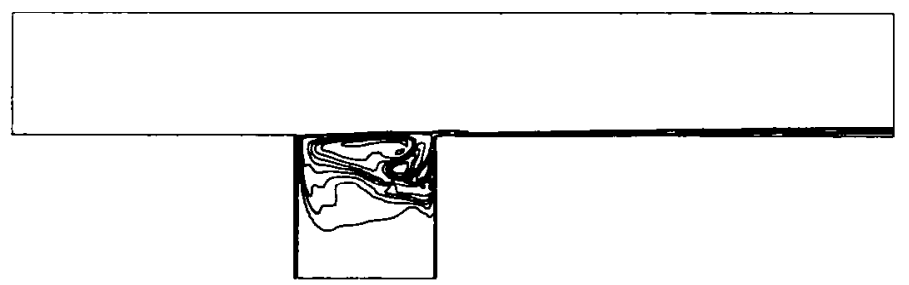

FiG. 5(a). Density contours for case (I) at $t=876 \mathrm{~s}$.

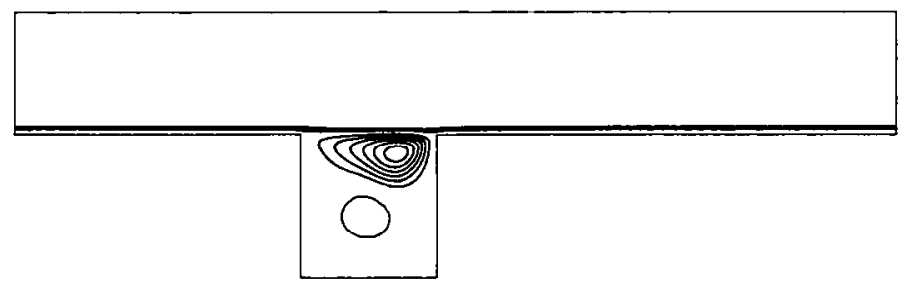

Fig. S(b). Stream function contours for case (1) at $t=876 \mathrm{~s}$.

rolls across the interface with a vortex that originates at the cavity inlet corner. The amplitude of the crest was observed to reduce with the Froude number and for case (4) was only just discernible.

Figure 6 shows a time series of the vertical velocity taken at a location 0.17 of the cavity width in from the inlet cavity wall and 0.78 of the cavity height up from the cavity bottom, for case (1). There is a noticeable oscillation in the signal, which decays rapidly. The period of the oscillation initially increases rapidly, but then becomes constant with a period of approximately $681 \mathrm{~s}$. Figure 7 is a time series of the Nusselt number calculated on a vertical line across the duct directly downstream of the cavity, for the early part of the flow. The Nusselt number, defined

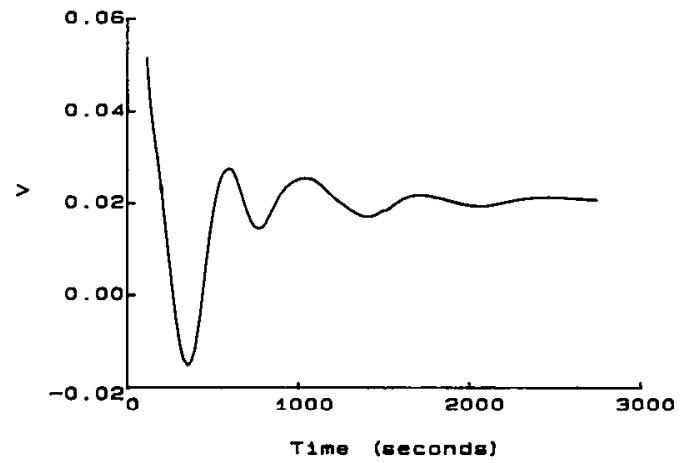

FIG. 6. Time series of vertical velocity in the cavity at $x=0.17$ of the cavity width from the cavity inlet wall and $y=0.78$ of the cavity height from the cavity bottom, for case (1). 


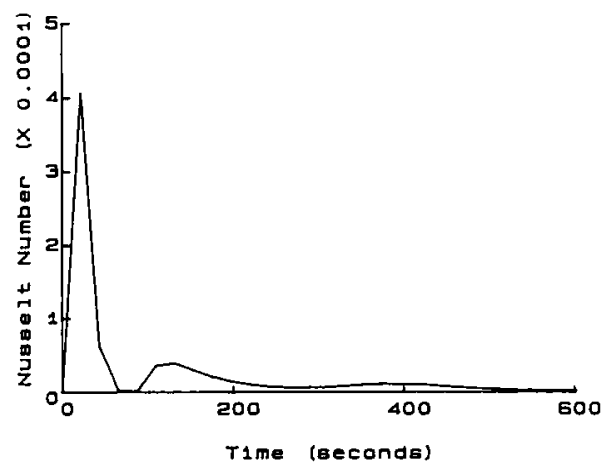

FIG. 7. Time series of Nusselt number on vertical line downstream of the cavity for case (1).

as

$$
N u=\frac{1}{2} \int_{\text {duct }}\left[\operatorname{Pr} U \sigma-\frac{\partial \sigma}{\partial x}\right] \mathrm{d} y
$$

is primarily a representation of the dense fluid advected out of the cavity by the overflow, as there is little diffusion. Three peaks are present in the Nusselt number plot, of decreasing magnitude, with the third being barely discernible. The initial, and most significant, peak is associated with the splash, while the second is associated with the crest striking the exit corner. The third peak is possibly associated with a seiching effect that will be discussed later.

Figure 8 is a time series of the Nusselt number at

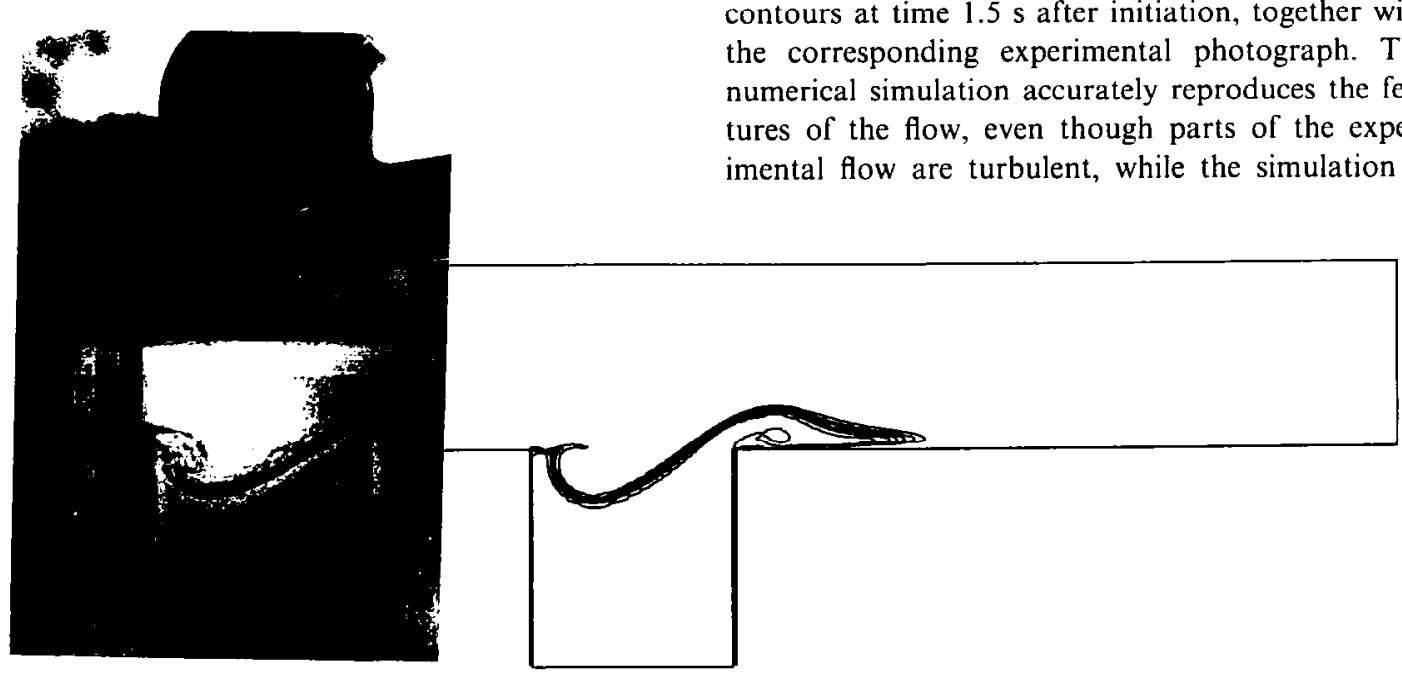

Fig. 9(a). Density contours for case (5) at $t=1.5 \mathrm{~s}$ with the corresponding experimental result.

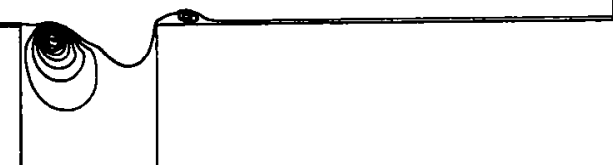

FIG. 9(b). Stream function contours for case (5) at $t=1.5 \mathrm{~s}$. 


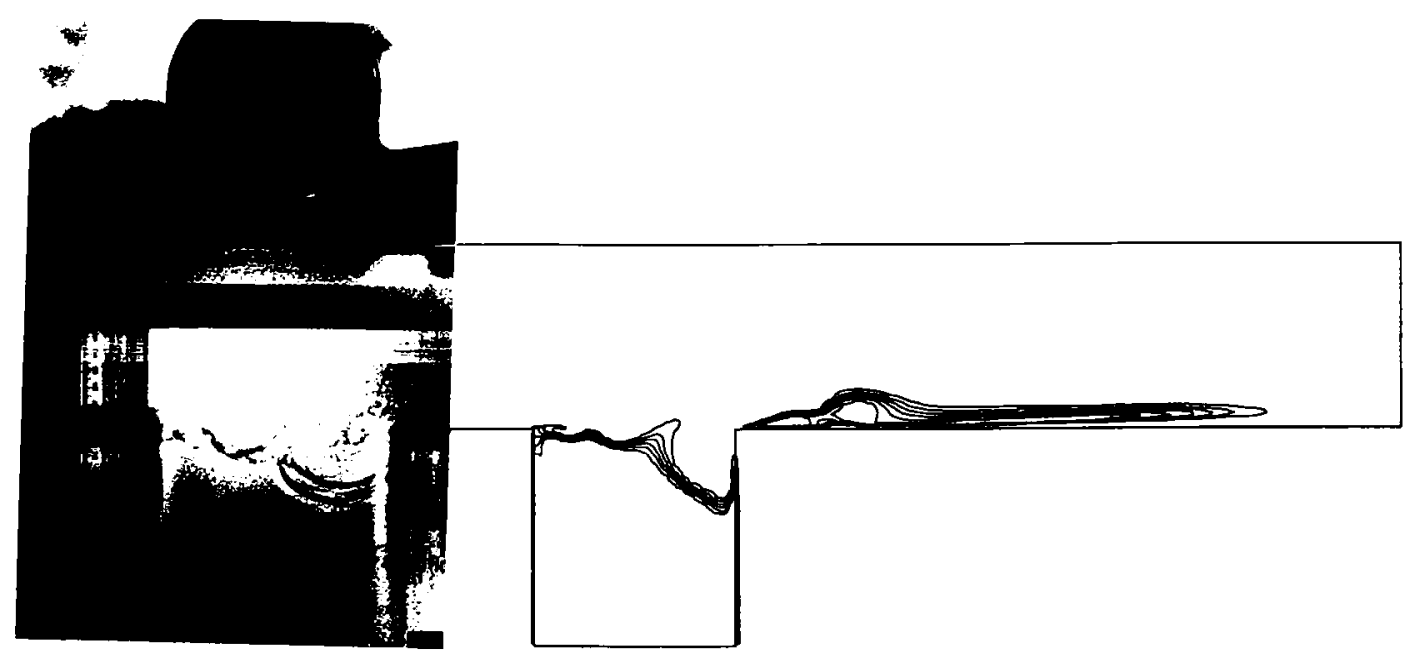

FIG. 10(a). Density contours for case (5) at $t=3.5 \mathrm{~s}$ with the corresponding experimental result.

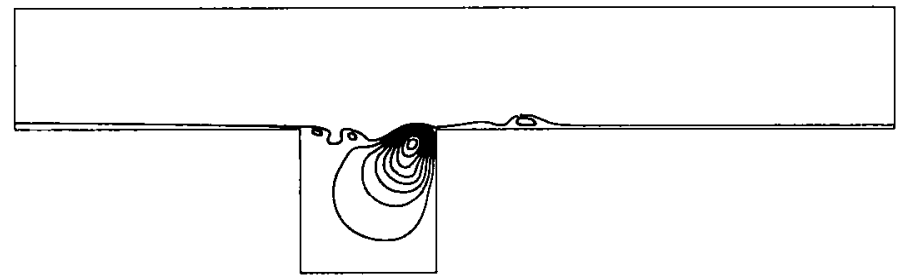

Fig. I0(b). Stream function contours for case (5) at $t=3.5 \mathrm{~s}$.

laminar. Once again a large splash of fluid is pushed out of the cavity by the overflow while a crest forms at the inlet corncr. The strcamlincs presented in Fig. 9(b) show that the crest is associated with a vortex that has formed at the inflow corner of the cavity, while the splash is associated with streamlines that pass from the overflow into the cavity and then out again at the downstream corner, similar behaviour to that observed in case (1) above.

By time $t=3.5 \mathrm{~s}$ after initiation, it is seen in the density contours shown in Fig. 10(a) that the crest has travelled across the cavity and is about to strike the far wall. It is also seen that additional smaller crests have formed behind the first crest. In the experimental result (also shown in Fig. 10(a)) there is an indication of additional crests, although the strong mixing makes them difficult to discern. Once again there is a good correspondence between the simulation and the experiment. The streamlines at this time, presented in Fig. 10(b), show that the main vortex associated with the initial crest is now centred near to the exit wall of the cavity. Additional smaller vortices associated with the additional crests are also visible.

In the further development of the flow at $t=4.5 \mathrm{~s}$, shown in Fig. 11, it is seen that the initial crest has struck the exit wall of the cavity and engulfed a volume of lighter fluid. The two subsequent crests have continued to travel across the cavity. Once again this is a good representation of the experimental results, although the subsequent crests are again not easily discernible in the experiment. The streamlines show that the initial vortex is now much contracted, and is associated fully with the engulfed volume of light fluid. It is also clear that the subsequent crests are associated with small vortices.

In Fig. 12(a), $t=61 \mathrm{~s}$ after initiation, it is seen that a mixed layer has formed, with a well defined interface between it and the unmixed dense fluid remaining in the cavity. The interface and mixed region has a second mode structure. The streamlines (Fig. 12(b)) show that two counter-rotating vortices are located in the mixed layer region, while a single vortex is located in the unmixed denser fluid. The two vortices in the mixed layer region are characteristic of a second mode internal wave, while at this stage a cavity scale vortex has developed in the unmixed fluid.

In Fig. 13 time series of the vertical velocity in the cavity at twe locations are shown. One location is 0.086 of the cavity width in from the inlet wall and 0.78 of the cavity height above the floor, while the other is at the same height but on the centreline of the cavity. As can be secn, both signals show a regular oscillation approximately $180^{1}$ out of phase, with a period of $6 \mathrm{~s}$. A node with minimum amplitude has been found to lie between the two locations shown which, taken together with the phase shift, indicates that the signal is produced by a second mode internal wave. 


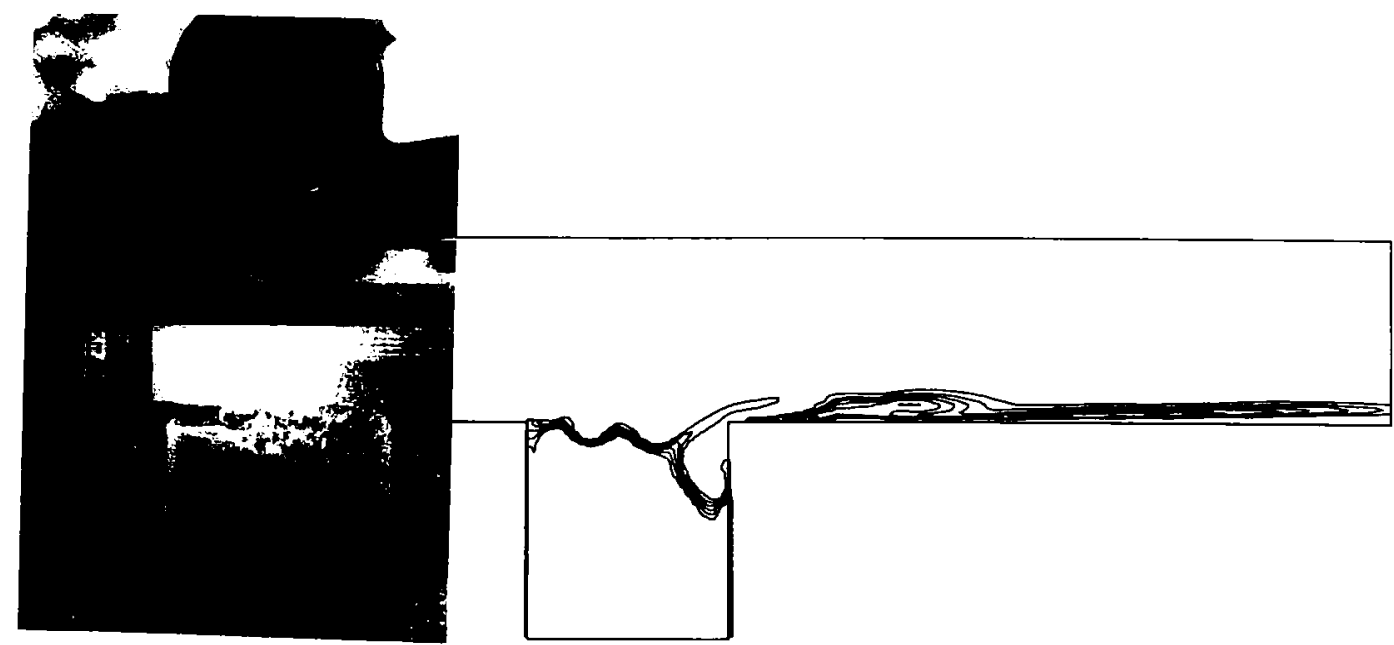

FIG. I1(a). Density contours for case (5) at $t=4.6 \mathrm{~s}$ with the corresponding experimental result.

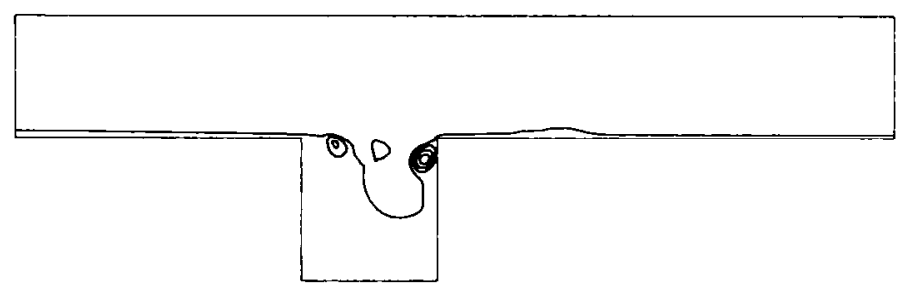

FIG. 11 (b). Stream function contours for case (5) at $t=4.6 \mathrm{~s}$.

Figure 14 shows a time series of the Nusselt number. As before this is primarily a measure of the advection of dense fluid out of the cavity, and once again it is apparent that the advection is dominated by the initial splash, represented by the first peak in the plot, followed by the advection resulting from the initial crest striking the exit wall of the cavity, represented by the second crest. Figure 15 shows the Nusselt number on an enlarged vertical scale for the full development of the flow. A regular oscillation is seen with the same period as that of the second mode signal observed above in the velocity time series (Fig. 13).

\section{DISCUSSION}

The results presented in the previous section indicate that the dominant features in the early part of the flow development are the initial splash and the

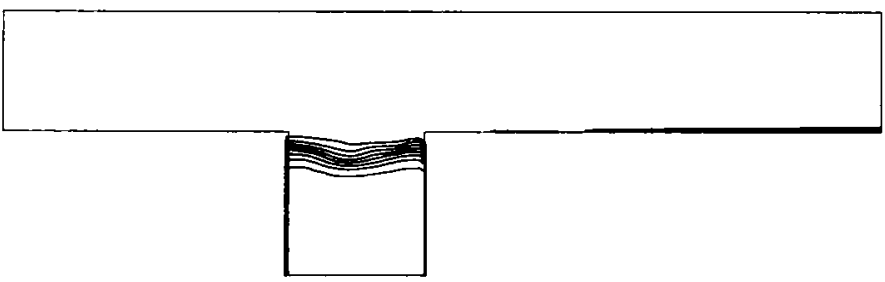

FIG. 12(a). Density contours for case (5) at $t=61 \mathrm{~s}$.

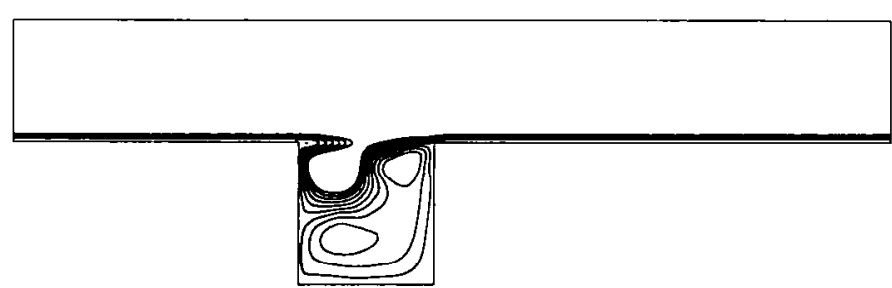

FIG. 12(b). Stream function contours for case (5) at $t=61 \mathrm{~s}$. 


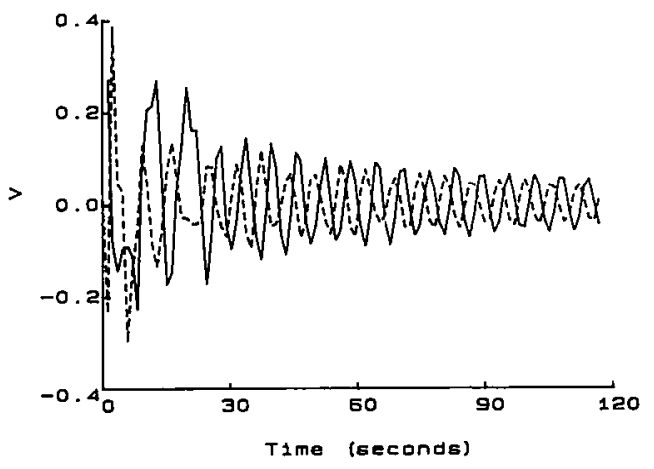

Fig. 13. Time series of vertical velocity in the cavity at $x=0.086$ of the cavity width from the cavity inlet wall and $y=0.078$ of the cavity height from the cavity bottom (solid line) and the same height and half the cavity width (dashed line), for case (5).

crest travelling across the cavity and associated secondary splash. Of these two features the initial splash contributes by far the largest component to the time rate of transport of dense fluid from the cavity into the duct. The initial splash is generated by the impulsive start-up of the system in the following way.

Immediately after start-up and away from the entrance the fluid responds inviscidly. Thus the velocity field in the cavity will be that of potential flow with a line source at the entrance and a line sink at the exit. This is readily seen in the results where, in Fig. 2(b), streamlines are observed passing from the overflow into the cavity and out again. The resulting velocity imparted to the fluid by the impulsive startup is enough to carry some of the dense fluid out of the cavity. It is this fluid that forms the initial splash.

This hypothesis may be used to construct an approximate relation for the drop in the level of the interface resulting from the fluid ejected from the cavity in the splash. It seems reasonable to assume that $\delta$, the interface drop non-dimensionalized by the cavity height, will be a function of the cavity Froude number, giving

$$
\delta \sim k F r
$$

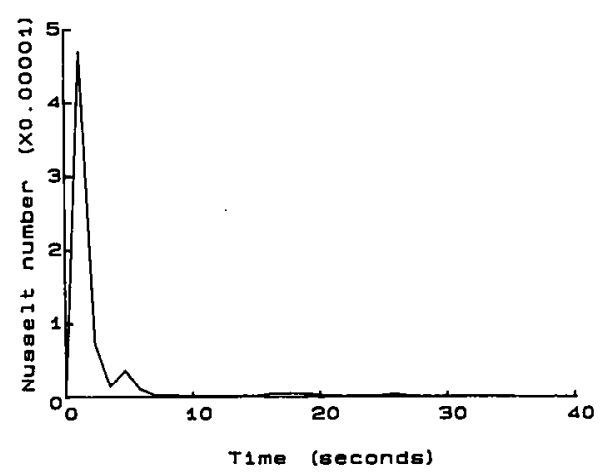

FIG. 14. Time series of Nusselt number on vertical line downstream of the cavity for case (5).

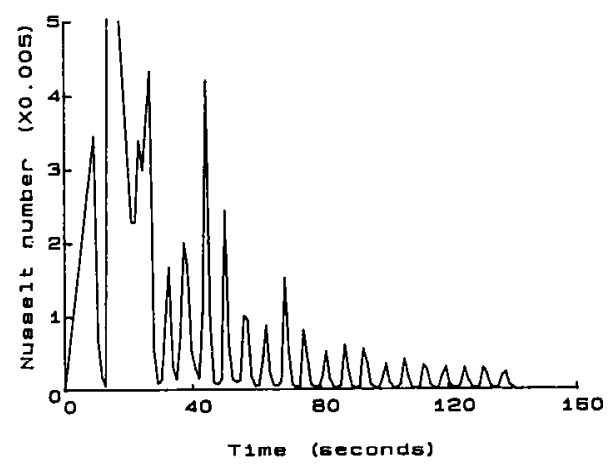

FIG. 15. Time series of Nusselt number with enlarged vertical scale on vertical line downstream of the cavity for case (5).

where $k$ is a constant and $F r$ is the cavity Froude number as defined in Section 3. The linear assumption cannot be true for all $F r$, as it would imply that for large enough $F r, \delta$ could be greater than the cavity height. Thus, although for the present range of Froude numbers considered the linear assumption gives good results, a more complex relation would have to be developed to cover all possible Froude numbers.

The six cases presented in this paper have interfacial drops resulting from the initial splash as follows:

Case (1) $\delta=0.43$
Case (2) $\delta=0.23$
Case (3) $\delta=0.28$
Case (4) $\delta=0.11$
Case (5) $\delta=0.09$
Case (6) $\delta=0.14$

Figure 16 shows $\delta$ plotted against $F r$. As can be seen, the points fall approximately on a straight line, giving $k=0.066$. Further points will need to be obtained for different aspect ratio cavities to verify this scaling formula; however, as a rule of thumb indicator for the square cavity it appears to be satisfactory.

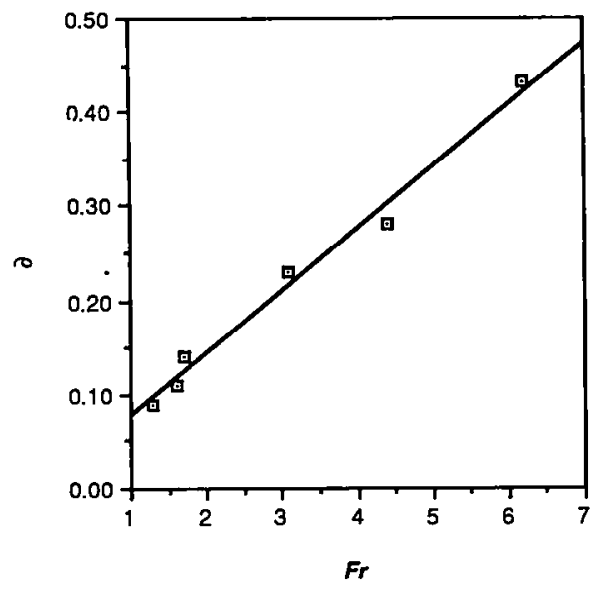

Fig. 16. Plot of interfacial depth $\delta$ against $F r$ for cases (1)-(6). 
The second feature, the crest. which contributes a much smaller amount to the purging of the cavity than does the splash, is associated with the formation of a vortex in the following way. At start-up a vortex sheet lies along the bottom of the duct which, as the flow develops, diffuses in the normal way to produce a vortex layer. Thus initially the inlet corner of the cavity is a point source of vorticity, which is subsequently transported across the cavity by the overflow. The point source of vorticity enlarges as the vorticity boundary layer develops, resulting in the development of the observed vortex. The point source lies initially at the interface, and so as it diffuses it entrains both light and heavy fluid. The entrained heavy fluid forms the resulting crest. The vortex-crest structure is then advected across the cavity by the overflow.

When the vortex strikes the exit corner the crest is high enough so that it at least partly passes out of the cavity, resulting in the second peak seen in the Nusselt number trace. The remaining dense fluid in the crest has not been lifted high enough to exit the cavity, and subsequently drops back down, engulfing a volume of lighter fluid. The dense and light fluid are then mixed by the vortex. The remainder of the dense fluid in the cavity is too stable to be fully turned over, and thus the initial cavity scale vortex contracts to fill only the upper part of the cavity. In the remainder of the cavity a weak counter-rotating vortex forms.

In the higher Reynolds number flows additional small crests and associated vortices were observed behind the main crest. It is suggested that these are the result of a Kelvin-Helmholtz type instability forming on the vortex sheet. For the lower Reynolds number the wavelength of such an instability was long enough so that it was not observed.

The features described above occur early in the development of the flow and contribute by far the major component to the rate of efflux of dense fluid from the cavity. After the passage of the crest and the second splash an oscillatory signal is present in the velocity time series, as shown in the previous section. The velocity signal has a decaying amplitude and, in the later part of the flow development, an approximately constant period. This oscillation is most likely the result of a cavity scale seiche. The period of such a seiche may be obtained from the formula [5] :

$$
p=\lambda\left[\frac{\Delta \rho g}{k}\left(\rho_{\mathrm{c}} \operatorname{coth} k h_{\mathrm{c}}+\rho_{\mathrm{d}} \operatorname{coth} k h_{\mathrm{d}}\right)^{-1}\right]^{-1 / 2} .
$$

For case (1) this gives a period for the tirst mode wave (wavelength $(\lambda)=2 h_{\mathrm{c}}, k=\pi / h_{\mathrm{c}}$ ) of $126 \mathrm{~s}$. For case (5) the period of the second mode internal wave is obtained from the above relation as $p=5.4 \mathrm{~s}$. The prediction for case (5) is in good agreement with the observed period, while that for case (1) is poor. It is likely that in case (1) the fluid is far from the twolayer form on which the above equation is based.
Additionally the relatively short data set (with respect to the period) and the rapid decay combine to make accurate measurement of the period difficult. For case (5) the data set is long with respect to the period and the decay is small which, combined with the reasonable two-layer structure observed in the density contours in the previous section, suggest that if the observed wave is a seiche the above equation should be a good predictor, as is the case.

The oscillations in the Nusselt number trace for the full development of the flow clearly have the same period as the velocity signal, and therefore it is suggested that they are the result of the seiche bringing denser fluid up to the overflow, where it is subsequently advected away. There is an indication of a possible additional mode in the lower Reynolds number case, which is most likely the result of, initially, dense fluid being brought up by both the forward and backwards seiche. There is also some indication of another mode in the higher Reynolds number signal; however, there the dominant signal is clearly that of the second mode wave acting at the exit wall of the cavity.

After the features described above have occurred the interface continues to drop until finally the entire cavity is purged. This later part of the flow development has not yet been studied in detail numerically; however, the experiments have been conducted and give a good indication of the method of purging the remainder of the cavity. The vortex that is located in the mixing region continues to be driven by the overflow. At the same time turbulent mixing occurs at the interface. The resulting fluid is transported to the mouth of the cavity by the vortex, where it is advected downstream. This process produces a much smaller efflux of fluid than the features described above, and thus the purging of the remainder of the cavity is relatively slow.

\section{CONCLUSIONS}

A comparison of experimental and numerical results has demonstrated that the early part of the purging of a density stabilized basin can be qualitatively well predicted using the finite-volume scheme described in Section 2. The numerical scheme has been used to produce results for a range of parameter values to allow the dominant parameter independent features of the flow to be identified and studied in detail. The existence of similar features over the range of values tested indicates they are not grid-dependent effects.

The early part of the flow has been shown to be dominated by the development of a splash resulting from the impulsive start-up. This short-lived feature carries a substantial amount of dense fluid out of the cavity, producing a time rate of efflux two orders of magnitude greater than any other feature in the flow, resulting in a sudden lowering of the interface. The amount of fluid ejected by the splash has been related 
to the internal cavity Froude number via the cavity length scale. The accurate results achieved by this approximation indicate that it is a good predictor for the square cavity; however, additional testing is required at different aspect ratios.

Subsequent to the splash a crest travels across the cavity mouth resulting in, when it strikes the far wall of the cavity, a second, albeit much smaller, peak in the efflux of dense fluid. The crest is associated with the development of a vortex layer on the floor of the duct and is formed by the rolling up of the interface in a vortex that is generated at the inlet corner of the cavity and then travels across the interface. At the higher Reynolds number additional crests were observed of a smaller amplitude behind the main crest. It is suggested that these may be the result of a KelvinHelmholtz type instability of suitable length scale.

After the effluxes caused by the splash and crest the internal wave is the major modulating factor on the Nusselt number. Thus the seiching carries dense water up to the mouth of the cavity where it is advected away by the overflow. As a result of this process the modal activity observed in the cavity is also observed in the Nusselt number.

The internal waves ultimately die out and the remainder of the cavity is then purged by mixed fluid being transported to the top of the cavity by a vortex that is located in the mixing region. This later period of purging is poorly predicted by the present scheme, most probably because the mixing is then dominated by small scale effects that are not accurately resolved. It is suggested that a large eddy simulation schemc is necessary to satisfactorily predict the later part of the flow. Such a scheme is currently under development.

Acknowledgements-The authors wish to thank Bill Deugd for assistance with building and operating the experimental equipment, and John Patterson and John Taylor for their useful suggestions. This research was supported by the Australian Research Council in the form of a National Research Fellowship and by grant A48615453, by the Australian Water Research Advisory Council, by the Victorian EPA and by a University of Western Australia Gleddon Visiting Lectureship.

\section{REFERENCES}

I. J. R. Anderson and A. K. Morison, Environmental consequences of saline groundwater intrusion into the Wimmera River, Victoria, BMR J. Aust. Geol. Geophys. 11, 233-235 (1989).

2. P. H. Gaskell and K. C. Lau, Curvature compensated convective transport: smart, a new boundedness preserving transport algorithm, Int. J. Numer. Meth. Fluids 8, 617-641 (1988)

3. B. P. Leonard, A stable and accurate convective modelling procedure based on quadratic upstream interpolation, Comput. Meth. Appl. Mech. Engng 19, 59-98 (1979).

4. S. W. Armfield, Finite difference solutions of the NavierStokes equations on staggered and non-staggered grids. Comput. Fluids 20, 1-17 (1991).

5. S. Turner, Buoyancy Effects in Fluids. Cambridge University Press, Cambridge (1979). 